\title{
BILATERAL PREHILAR BRANCHING OF RENAL ARTERIES
}

\section{Anuj Ram Sharma ${ }^{1}$, Rakesh Kumar Agarwal *2, Hemant Saini ${ }^{3}$, Seema Rani Khajuria ${ }^{4}$, Anil Kumar Malik ${ }^{5}$.}

${ }^{1}$ Assistant Professor, Department of Anatomy, Muzaffarnagar Medical College and Hospital, Muzaffarnagar (U.P.), India.

${ }^{* 2}$ Assistant Professor, Department of Anatomy, Maharishi Markandeshwar Medical College and Hospital, Kumarhatti, Solan (H.P.), India.

${ }^{3}$ Assistant Professor, Department of Anatomy, Maharishi Markandeshwar Medical College and Hospital, Kumarhatti, Solan (H.P.), India.

${ }^{4}$ Assistant Professor, Department of Anatomy, Maharishi Markandeshwar Medical College and Hospital, Kumarhatti, Solan (H.P.) India.

${ }^{5}$ Assistant Professor, Department of Forensic Medicine, Maharishi Markandeshwar Medical College and Hospital, Kumarhatti, Solan (H.P.), India.

\section{ABSTRACT}

Background: Variation in renal arteries are very common but bilateral variation is rare and is clinically important for urologist especially during the renal transplantation. Generally main renal artery divides into anterior and posterior branches just before entering the hilum of kidney, but sometimes two or more polar arteries; prehilar branching and segmental arteries arise from main renal artery . Renal artery variations are often seen and are generally categorized into presence of accessory or aberrant renal arteries, polar arteries and prehilar branches. Prehilar multiple branching of main renal artery variation is frequently seen.

Materials and Method: The present study was conducted in 80 kidneys from 40 cadavers during the routine dissection in the Department of Anatomy Maharishi Markandeshwar Medical College and Muzaffarnagar Medical College .Out of 80 kidneys studied bilateral prehilar branching of renal arteries from the main renal artery were observed in 5 kidneys (6.5\%).

Conclusion: Different arterial branching patterns was observed in five cases in which prehilar branches originated directly from main renal artery, entering the upper pole of kidney. Knowledge of such variation is important for radiologists, anatomists and urologists while performing renal transplantation, laparoscopic renal surgeries, nephrectomies, and other renal surgeries and diagnosis.

KEY WORDS: Upper Polar Artery, Renal Artery Variations, Segmental Artery, Main Renal Artery, Bilateral Prehilar Branching.

Address for Correspondence: Dr. Rakesh Kumar Agarwal, Assistant Professor, Department of Anatomy, Maharishi Markandeshwar Medical College and Hospital, Kumarhatti Solan-173229 (H.P.) India. E-Mail: drrakeshagarwal@rediffmail.com

Access this Article online

Quick Response code

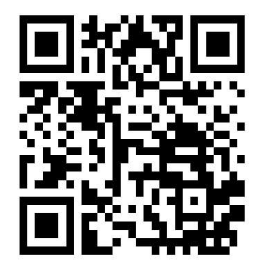

DOI: $10.16965 /$ ijar.2018.263

Journal Information

International Journal of Anatomy and Research

\begin{tabular}{|c|c|} 
ICV for 2016 \\
$\mathbf{9 0 . 3 0}$
\end{tabular}$\quad \begin{gathered}\text { ISSN (E) 2321-4287 I ISSN (P) 2321-8967 } \\
\text { https://www.ijmhr.org/ijar.htm } \\
\text { DOI-Prefix: https://dx.doi.org/10.16965/ijar }\end{gathered}$

Article Information

Received: 26 May 2018

Peer Review: 27 May 2018

Revised: None
Accepted: 02 Jul 2018

Published (O): 10 Aug 2018

Published (P): 10 Aug 2018

\section{INTRODUCTION}

Normally a single renal artery supplies each kidney arising as a lateral branch of abdominal aorta below the superior mesenteric artery as a 
$5^{\text {th }}$ branch at the level of L1-L2. It runs obliquely and laterally downwards toward the hilum of kidneys and divides into anterior and posterior branches in reference with renal pelvis.

Renal artery variations are often seen and are generally categorized into presence of accessory or aberrant renal arteries, polar arteries and prehilar branches. Budhiraja et al observed Prehilar multiple branching of main renal artery variations in $11.6 \%$ of cases[1]. Shoja et al and Rao et al observed duplicate, triplicate, fork pattern and ladder pattern in Prehilar multiple branching in their studies[2, 3].

Budhiraja et al and Gray \&Skandalakis et al observed in their studies that origin of superior polar artery from main renal artery was present in $5.4 \%$ of cases [4] and $12.6 \%$ of cases, respectively [5].

Surgeons and radiologists should be aware of such variations of renal vasculature while performing renal transplantation, laparoscopic renal surgeries, nephrectomies, and other renal surgeries and diagnosis.

\section{MATERIALS AND METHODS}

The present study was conducted on 80 kidneys harvested from 40 cadavers during routine anatomical dissection in the Department of Anatomy Maharishi Markandeshwar Medical College and Hospital Kumarhatti, Solan H.P. and Muzaffarnagar Medical College and Hospital Muzaffarnagar U.P. The kidneys and their vasculature were carefully explored and variations in morphological pattern of renal arteries were noted. Renal veins and Inferior vena cava were also reflected for proper visualization of pattern of renal arteries.

\section{OBSERVATIONS AND RESULTS}

In our study conducted on 80 kidneys, we observed that in 5 kidneys ( $6.5 \%$ of cases), bilateral prehilar branching of renal arteries originated from the main renal artery. In all five cases separate segmental artery (prehilar branch) were noted originating from main renal artery. These arteries originated from renal artery directly and enter the renal parenchyma of upper pole of kidney from anteromedial side to supply upper pole of the kidney(fig.1,fig.2).
Fig. 1: Right and left kidney showing bilateral prehilar branch of renal arteries.

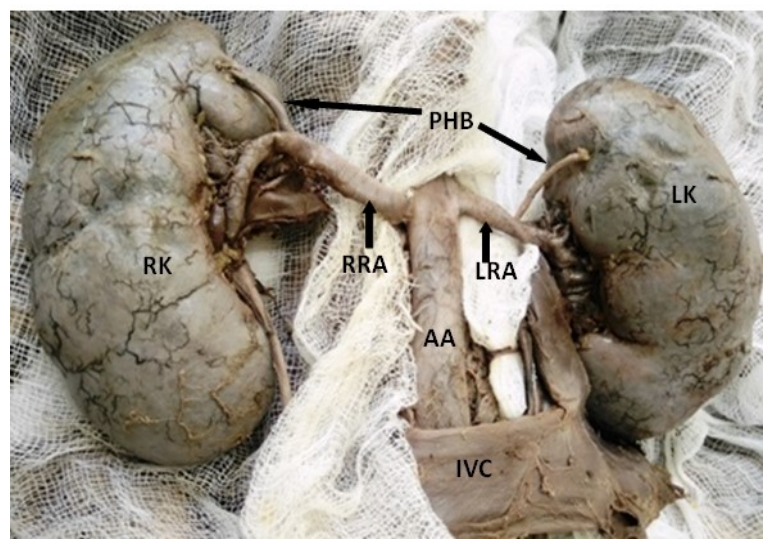

(RK-Right kidney, LK-Left kidney, PHB-prehilar branch , RRA-Right Renal artery, LRA- Left renal artery, Abdominal aorta, IVC-Inferior vena cava).

Fig. 2: Renal arteries showing bilateral prehilar branches.

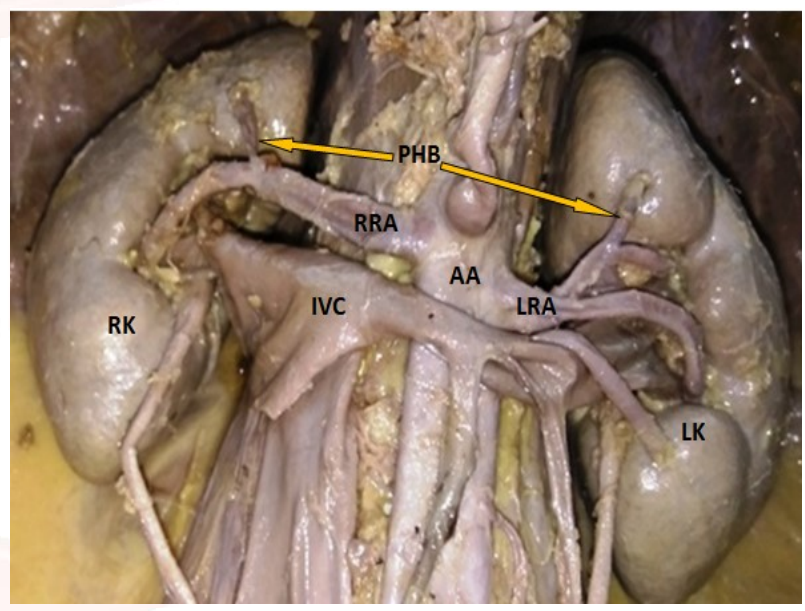

(RK-right kidney, LK-left kidney, RRA-right renal artery, LRA-left renal artery, PHB - prehiliar branch, IVC- inferior vena cava, AA-abdominal aorta)

\section{DISCUSSION}

In our study, the incidence of origin of bilateral prehilar segmental branch from renal artery is $6.5 \%$, which is in concordance with the study by Rusu et al [6]. Characteristically, in all of the cases studied by us, the upper polar artery arise from the main renal artery and enters the renal parenchyma directly.

Kaneko et al. in their study, observed that 36 cases (21.2\%) had multiple arterial origins either on the left or right side, and 8 cases $(4.7 \%)$ had bilateral multiple arterial origins[7].

K S Satyapal et al.studied 130 angiograms and 32 cadavers, where they observed that $23 \%$ of cases had double renal artery on right side, followed by left side in $32 \%$ of cases and bilaterally in $10 \%$ of cases [8]. So, bilateral segmental 
branch of renal artery is a rare variation. Bordei et al. conducted study on about 272 kidneys and found bilateral double renal artery in only $2.2 \%$ of cases [9]. Virendra Budhiraja et al. studied about 100 kidneys and found bilateral double renal artery in only $11.6 \%$ of cases [10]. They also stated that duplicated renal arteries is result of persisting lateral mesonephric arteries from middle group.

Albohassan while studying angiography in renal transplant donor observed less than $1 \%$ incidence of bilateral double renal artery [11].

Bayramoglu et al. reported a variant which consisted of bilateral additional renal arteries originating from the abdominal aorta and an additional right renal vein accompanying the additional right renal artery. These anomalies were associated with unrotated kidneys with extrarenal calices and pelvis. All the additional vessels were located posterior to the ureter with a close relationship to the ureteropelvic junction on the right side [12].

Gray \&Skandalakis et al. observed origin of superior polar artery from the main renal artery in $12.6 \%$ of cases [5].

Saldarriaga et al. observed origin of superior polar artery from renal artery in $17.2 \%$ of cases on the right side and $13.5 \%$ of cases on the left side [13]. Budhiraja et al. reported the origin of superior polar artery from main renal artery in $5.4 \%$ of cases [4].

Rao et al observed variations of renal arteries. In their study they observed that the branches were directed towards superior, middle, inferior and posterior vascular segments of kidney [3]. As upper polar branches of renal artery correspond to segmental arteries, it increases the risk of hemorrhage during renal transplantation, segmental ischemia and postoperative hypertension due loss of parenchyma. The surgical accessibility to clamping of segmental arteries from anterior and posterior approaches was determined by Weld et al[14].

There are few reports of the origin of the upper polar artery from the segmental branch by Bakheit et al and Rao et al[15,3]. In their studies, they observed that arteries run vertically before entering the kidney. According to the Beyer et al, such vertically directed upper polar (superior polar) arteries cause upper pole infarction. Near the hilum of the kidney each renal artery divides into an anterior and posterior branch, which in turn divides into segmental branches in the renal sinus or prior to the entrance through the hilum [16].

The hilar pattern of segmental branches of the renal arteries had been discussed previously by Shoja et al. and Weld et al $[2,14]$. Nayak et al described a case in which three renal arteries provided seven segmental branches, supplying one kidney. Among the seven segmental branches, two entered the kidney by piercing through its anterior surface, and the remaining five entered through the hilum [17].

Rusu et al mentioned double right hilar renal arteries disposed side by side, the superior hilar and inferior hilar, both dividing into two segmental branches each, which were arranged anterior and posterior to the renal pelvis, respectively [6]. We also observed upper polar branching patterns of the renal artery. In most of the cases these segmental branches arise from the main renal artery at some distance before it reached the hilum.

Shamilla et al observed that pre-segmental branches originated from the main renal artery in $13.4 \%$ of cases. These entered either through the upper pole or lower pole as polar artery [18]. Bordei $P$ et al observed in their study that superior polar artery originated from main renal artery in $9.2 \%$ of cases [9].

\section{CONCLUSION}

Bilateral prehilar branching of renal arteries or upper polar artery is a segmental artery supplying the upper pole of the kidney. Its origin from the main renal artery as an upper polar branch is clinically important because inadvertent damage of this segmental artery during renal transplant surgeries, laproscopic renal surgeries, nephrectomies and other renal procedures will produce infarction of the superior segment of the kidney. Knowledge of such variations is important for radiologists, anatomists and urologists.

\section{Conflicts of Interests: None}




\section{REFERENCES}

[1]. Budhiraja V, Rastogi R,Asthana A K. Renal artery variations: embryological basis and surgical correlation.Romanian Journal of Morphology and Embryology, 2010;51(3):533-36.

[2]. Shoja MM, Tubbs RS, Shakeri A, Loukos M, Ardalan MR, Khosroshahi HT, Oakes WJ. Perihilar branching patterns and morphologies of renal artery: a review and anatomical study. Surg Radiol Anat, 2008;30:375-82.

[3]. Rao M, Bhat SM, Venkataramana V, Deepthinath R, BollaSR . Bilateral prehilar multiple branching of renal arteries: A case report and literature review. Kathmandu Univer Med J,2006;4:345-48.

[4]. Budhiraja V, Rastogi R, Asthana A K . Variant origin of superior polar artery and unusual hilar branching pattern of renal artery with clinical correlation. Folia Morphol.2011;70(1):24-28.

[5]. Gray, S. W., and Skandalakis, J. E. Anomalies of the kidney and ureter. In Embryology for Surgeons. Philadelphia, W. B. Saunders Co.1972; p. 485.

[6]. Rusu MC.Human bilateral doubled renal and testicular arteries with a left testicular arterial arch around the left renal vein. Rom J Morphol Embryol,2006; 47: 197-200.

[7]. Kaneko N, Kobayashi Y, Okada Y. Anatomic variations of the renal vessels pertinent to transperitoneal vascular control in the management of trauma. Surgery. 2008;143: 616-22.

[8]. Satyapal KS ,Haffejee AA et al. Additional renal arteries; incident and morphometry,Surg radiol Anat, 2001;23;33-38.

[9]. BordeiP,Sapte E et al,Double renal arteries originating from aorta,Surg Radiol Anat 2004;26;474-79.
[10]. Virendra Budhiraja et al, Renal artery variation: embryological basis and surgical correlation Romanian journal of morphology and embryology ,2010; 51(3);533-36.

[11]. Alboo Hssan,Shakeri et al, Bipolar supernumerary renal artery: surgical and radiological anatomy, Anatomic variation 2007;29:89-92.

[12]. Bayramoglu A, Demiryurek D, Erbil KM. Bilateral additional renal arteries and an additional right renal vein associated with unrotated kidneys. Saudi Med J. 2003;24: 535-37.

[13]. Saldarriaga B, Pinto SA, Ballesteros LE. Morphological expression of the renal artery. A direct Anatomical study in a Colombian half-caste population. Int J Morphol,2008; 26: 31-38.

[14]. Weld KJ, Bhayani SB, Belani J, Ames CD, Hruby G, Landman J. Extrarenal vascular anatomy of kidney: assessment of variations and their relevance to partial nephrectomy. Urology,2005; 66: 985-89.

[15]. Bakheit MA, Motabagani MA. Anomalies of the renal, phrenic and suprarenal arteries: case report. East Afr Med J,2003; 80: 497-500.

[16]. Beyer RW, Daily PO. Renal artery dissection associated with GZ acceleration. Aviat Space Environ Med, 2004;75: 284-87.

[17]. Nayak BS.Multiple variations of the right renal vessels. Singapore Med J,2008; 49: e153-55.

[18]. Sharmila A Sundarapandian, christilda F. Anatomical Study of Variations in Blood Supply of Kidneys. J ClinDiagh Res 2013 Aug;7 (8): 1555- 57.

How to cite this article:
Anuj Ram Sharma, Rakesh Kumar Agarwal, Hemant Saini, Seema Rani
Khajuria, Anil Kumar Malik. BILATERAL PREHILAR BRANCHING OF RENAL
ARTERIES. Int J Anat Res 2018;6(3.2):5511-5514. DOI: 10.16965/
ijar.2018.263 